\title{
Rotating Scheimpflug Imaging Indices in Different Grades of Keratoconus
}

\author{
Sherine S. Wahba, ${ }^{1,2}$ Maged M. Roshdy, ${ }^{1,2}$ Rania S. Elkitkat, ${ }^{1,2}$ and Karim M. Naguib ${ }^{1,2}$ \\ ${ }^{1}$ Faculty of Medicine, Ain Shams University, Cairo 11566, Egypt \\ ${ }^{2}$ Al Watany Eye Hospitals, 211 Alhegaz Street, Alhegaz Square, Heliopolis, Cairo 11361, Egypt
}

Correspondence should be addressed to Rania S. Elkitkat; raniaselkitkat@med.asu.edu.eg

Received 9 March 2016; Revised 26 June 2016; Accepted 10 July 2016

Academic Editor: Sang Beom Han

Copyright ( $(2016$ Sherine S. Wahba et al. This is an open access article distributed under the Creative Commons Attribution License, which permits unrestricted use, distribution, and reproduction in any medium, provided the original work is properly cited.

\begin{abstract}
Purpose. To evaluate accuracy of various Keratoconus (KC) screening indices, in relation to Topographic Keratoconus (TKC) grading. Setting. Al Watany Eye Hospital, Cairo, Egypt. Methods. Data of 103 normal (group 1) and 73 KC eyes (group 2), imaged by Pentacam (branded as Allegro Oculyzer), were analysed. Group 2 was divided into $2 a$ : 14 eyes (TKC = 1, early KC), $2 b: 25$ eyes ( TKC $=1$ to 2 or 2 , moderate $\mathrm{KC}$ ), and $2 c: 34$ eyes (TKC $=2$ to 3 up to 4 , severe $\mathrm{KC}$ ). Participants were followed up for six years to confirm diagnosis. Area under the receiver operating characteristic curve (AUROC) was calculated for evaluated curvature, elevation, and pachymetry indices with various reference shapes at different diameters. Results. When comparing normal to KC eyes, ten indices had significantly higher AUROC. Only five of them had significantly higher AUROC in early KC compared to normal corneas: Pachymetry Progression Index- (PPI-) Maximum (Max), Ambrósio’s Relational Thickness- (ART-) Max, PPI-Max minus PPI-Minimum (Min), central corneal thickness (CCT), and diagonal decentration of thinnest point from the apex (AUROC $=0.690,0.690,0.687,0.683$, and 0.674 , resp.). Conclusion. Generally, ten pachymetry and elevation-based indices had significantly higher AUROC. Five indices had statistically significant high AUROC when comparing early KC to normal corneas.
\end{abstract}

\section{Introduction}

The hallmark characteristic of Keratoconus (KC) is the development of localized, cone-shaped ectasia accompanied by corneal thinning in the area of the cone. This leads to irregular astigmatism and steeper corneal curvature over the area of the cone [1]. The early detection of these changes is usually based on Placido-based corneal topography and rotating Scheimpflug camera corneal tomography. However, there are confounding conditions that can imply topographic and tomographic corneal changes resembling KC, such as corneal scarring, hyperopic refractive surgery, contact lens-induced warpage [2], and even normal variants of topographic curvature patterns.

There are several tomographic criteria for KC diagnosis. They can be divided into three main subgroups: curvaturebased, elevation-based, and pachymetry-based. Unfortunately, none of them is $100 \%$ sensitive and specific. Some authors believe that elevation maps are better than axial curvature maps for $\mathrm{KC}$ screening [3]. Others claim that curvature is still the most sensitive [4].

The rotating Scheimpflug camera "Pentacam" (Oculus $\mathrm{GmbH}$, Wetzlar, Germany) can generate various indices within each of the three indices subgroups.

Pentacam allows for measuring local elevation points by fitting the corneal shape to a best-fit sphere (BFS) reference surface with variable diameters or to a best-fit toric ellipsoid surface (BFTE), which simulates more the corneal shape than the BFS [5]. The guidelines differ between authorities. For instance, some prefer using BFS based on the analysis of $8 \mathrm{~mm}$ zone around the corneal apex, especially for refractive surgery screening $[6,7]$, others keep the device default at $9 \mathrm{~mm}$ zone, although there are some concerns about it; that is, it is more difficult to get reliable scans without wide palpebral fissure. On the contrary, BFTE has been recommended for use by other studies, as they concluded that toric ellipsoid reference 
surface is the most sensitive reference body to compare KC to normal corneas [8-10].

More recently, there have been other proposed indices; one of the most valuable $\mathrm{KC}$ indices is the Ambrósio's Relational Thickness (ART) [11].

This study aims at comparing the accuracy of each of the tested indices at various best-fit reference surfaces, in cohorts of $\mathrm{KC}$ and normal corneal cases who were followed up for six years, and to evaluate the accuracy of various indices in relation to Topographic KC (TKC) grading.

To the best of our knowledge, our study is innovative in evaluating the accuracy of $\mathrm{KC}$ indices using different reference surfaces of various diameters when applicable. Moreover, novelty lies in comparing the tested indices against time evidence, through our six-year follow-up interval (stability even after LASIK surgeries of normal corneas and spontaneous progression over years in $\mathrm{KC}$ cases). Time evidence is a definite proof of diagnosis not relying on subjective assessment.

\section{Patients and Methods}

A retrospective study was conducted using the data from consecutive patients' files that were imaged in the time interval between June 2008 and December 2009, using the Pentacam branded as Allegro Oculyzer (WaveLight, GmbH, Erlangen, Germany) [12] with software version 1.16r12 at Al Watany (National) Eye Hospital, Cairo, Egypt. Patients with any detected corneal pathology other than $\mathrm{KC}$ and patients with history of ocular surgery, contact lens wear during the previous two weeks, or narrow palpebral fissure impeding reliable imaging were excluded from the study. Any patient with questionable diagnosis at the time of initial Pentacam imaging was also excluded. Moreover, the patients were followed up until December 2015 to confirm the diagnosis and hence to confirm and evaluate the results.

Every eye was scanned at least thrice by the Allegro Oculyzer, in a dark room and according to the recommendations stated in the device manual. Each scan included 25 Scheimpflug images. Despite good repeatability, data were collected from the most reliable scan as stated by the "QS" pop-up box (i.e., largest analysed area, valid data percent, and good alignment). The data were collected from the automatically calculated indices or generated by manually changing the reference surface shape (BFS or BFTE) and calculation area $(7,8$, or $9 \mathrm{~mm})$ and getting the elevation values on mouse click. The investigated indices were as follows.

Curvature indices are as follows:

(i) Mean eccentricity of the anterior corneal surface in the central $3 \mathrm{~mm}$.

(ii) Mean radii of curvature of anterior and posterior corneal surfaces in the central $3 \mathrm{~mm}$ (in $\mathrm{mm}$ ).

(iii) Determinants of BFS:

(a) radii of curvature of the 8 and $9 \mathrm{~mm}$ anterior BFS (in $\mathrm{mm}$ ), (b) radii of curvature of the 7,8 , and $9 \mathrm{~mm}$ posterior BFS (in mm).

(iv) Determinants of posterior BFTE:

(a) eccentricity of the 7,8 , and $9 \mathrm{~mm}$ posterior BFTE,

(b) flattest and steepest radii of curvature of the 7, 8, and $9 \mathrm{~mm}$ posterior BFTE (in $\mathrm{mm}$ ).

Elevation indices are as follows:

(i) Elevation of the thinnest point from the $7 \mathrm{~mm}$ posterior (PE) BFS (in $\mu \mathrm{m}$ ).

(ii) Elevation of the thinnest point from the 8 and $9 \mathrm{~mm}$ anterior (AE) and posterior (PE) BFS and difference between corresponding anterior and posterior values (in $\mu \mathrm{m}$ ).

(iii) Elevation of the thinnest point from the 7,8, and $9 \mathrm{~mm}$ posterior (PE) BFTE (in $\mu \mathrm{m}$ ).

(iv) Maximum elevation, minimum elevation, and the difference between them (in $\mu \mathrm{m}$ ) from the 8 and $9 \mathrm{~mm}$ anterior BFS.

(v) Maximum elevation, minimum elevation, and the difference between them (in $\mu \mathrm{m}$ ) from the 7,8 and $9 \mathrm{~mm}$ posterior BFS.

(vi) Maximum elevation, minimum elevation, and the difference between them (in $\mu \mathrm{m}$ ) from the 7, 8 , and $9 \mathrm{~mm}$ posterior BFTE.

Pachymetry indices are as follows:

(i) Pachymetry at corneal apex, corneal center, and thinnest point (in $\mu \mathrm{m}$ ).

(ii) Pachymetry Progression Index-Average (PPI-Avg), Minimum (PPI-Min), and Maximum (PPI-Max) and the difference between PPI-Max and PPI-Min (PPIMax minus PPI-Min).

(iii) Ambrósio’s Relational Thickness-Average (ART-Avg), Minimum (ART-Min), and Maximum (ART-Max).

(iv) Horizontal and vertical decentration of the thinnest point from the apex and the resultant diagonal decentration $[=\sqrt{ }($ squared horizontal decentration + squared vertical decentration) (in $\mathrm{mm}$ )].

Patients were classified into two groups according to TKC staging adopted by the device software; group 1 included 103 normal eyes, while group 2 is comprised of 73 eyes with KC. Group 2 was furtherly divided into subgroups: group $2 a$ : 14 eyes $(\mathrm{TKC}=1$, early KC), group $2 b: 25$ eyes $(\mathrm{TKC}=1$ to 2 or 2 , moderate KC), and group $2 c: 34$ eyes (TKC $=2$ to $3,3,3$ to 4 , or 4 , severe KC).

The study adhered to the Tenets of the Declaration of Helsinki and to the local ethics standards. 
TABLE 1: The mean, SD, and 95\% confidence interval (CI) of all tested KC detecting parameters. Radii of curvature are in mm, elevations in $\mu \mathrm{m}$, pachymetry in $\mu \mathrm{m}$, and decentration in $\mathrm{mm}$.

\begin{tabular}{|c|c|c|c|c|c|c|}
\hline \multirow{2}{*}{ Indices } & \multicolumn{3}{|c|}{ Normal } & \multicolumn{3}{|c|}{ Keratoconus stages 1 to 4} \\
\hline & Mean & SD & $95 \% \mathrm{CI}$ & Mean & SD & $95 \% \mathrm{CI}$ \\
\hline \multicolumn{7}{|c|}{ Curvature indices } \\
\hline \multicolumn{7}{|l|}{ Central $3 \mathrm{~mm}$} \\
\hline Mean radius of curvature of anterior $3 \mathrm{~mm}$ corneal surface & 7.725 & 0.2305 & $7.323-8.149$ & 6.954 & 0.6640 & $5.296-8.124$ \\
\hline Mean radius of curvature of posterior $3 \mathrm{~mm}$ corneal surface & 6.425 & 0.256 & $6.040-6.809$ & 5.624 & 0.685 & $4.145-6.777$ \\
\hline Mean eccentricity of the anterior corneal surface & 0.519 & 0.132 & $0.210-0.730$ & 0.799 & 0.3312 & $-0.034-1.310$ \\
\hline \multicolumn{7}{|l|}{ Determinants of BFS } \\
\hline Radius of curvature of the $8 \mathrm{~mm}$ anterior BFS & 7.786 & 0.2252 & $7.413-8.200$ & 7.350 & 0.445 & $6.191-8.230$ \\
\hline Radius of curvature of the $9 \mathrm{~mm}$ anterior BFS & 7.833 & 0.2225 & $7.462-8.269$ & 7.488 & 0.398 & $6.535-8.284$ \\
\hline Radius of curvature of the $7 \mathrm{~mm}$ posterior BFS & 6.390 & 0.2147 & $5.991-6.758$ & 5.935 & 0.4148 & $5.109-6.700$ \\
\hline Radius of curvature of the $8 \mathrm{~mm}$ posterior BFS & 6.464 & 0.3544 & $6.031-6.790$ & 6.125 & 0.3842 & $5.465-7.022$ \\
\hline Radius of curvature of the $9 \mathrm{~mm}$ posterior BFS & 6.548 & 0.3426 & $6.101-6.899$ & 6.280 & 0.3041 & $5.765-6.981$ \\
\hline \multicolumn{7}{|l|}{ Determinants of BFTE } \\
\hline Flattest radius of curvature of the $7 \mathrm{~mm}$ posterior BFTE & 6.644 & 0.2495 & $6.143-7.152$ & 5.972 & 0.7057 & $4.312-6.947$ \\
\hline Steepest radius of curvature of the $7 \mathrm{~mm}$ posterior BFTE & 6.175 & 0.2317 & $5.741-6.609$ & 5.273 & 0.6972 & $3.971-6.630$ \\
\hline Eccentricity of the $7 \mathrm{~mm}$ posterior BFTE & 0.181 & 0.3173 & $-0.441-0.765$ & 0.618 & 0.5282 & $-0.447-1.350$ \\
\hline Flattest radius of curvature of the $8 \mathrm{~mm}$ posterior BFTE & 6.655 & 0.2507 & $6.143-7.169$ & 5.972 & 0.7057 & $4.312-6.947$ \\
\hline Steepest radius of curvature of the $8 \mathrm{~mm}$ posterior BFTE & 6.175 & 0.2317 & $5.741-6.609$ & 5.278 & 0.6936 & $3.971-6.630$ \\
\hline Eccentricity of the $8 \mathrm{~mm}$ posterior BFTE & 0.181 & 0.3173 & $-0.441-0.765$ & 0.618 & 0.5282 & $-0.447-1.350$ \\
\hline Flattest radius of curvature of the $9 \mathrm{~mm}$ posterior BFTE & 6.658 & 0.2665 & $6.143-7.169$ & 5.972 & 0.7057 & $4.312-6.947$ \\
\hline Steepest radius of curvature of the $9 \mathrm{~mm}$ posterior BFTE & 6.175 & 0.2317 & $5.741-6.609$ & 5.287 & 0.7092 & $3.971-6.630$ \\
\hline Eccentricity of the $9 \mathrm{~mm}$ posterior BFTE & 0.181 & 0.3173 & $-0.441-0.765$ & 0.618 & 0.5282 & $-0.447-1.350$ \\
\hline \multicolumn{7}{|c|}{ Elevation indices } \\
\hline \multicolumn{7}{|l|}{ Using BFS } \\
\hline Elevation of the thinnest point from the $7 \mathrm{~mm}$ posterior BFS & 1.5 & 3.13 & $-4.0-9.9$ & 46.2 & 32.7 & $2.3-115.4$ \\
\hline Maximum elevation from the $7 \mathrm{~mm}$ posterior BFS & 32.8 & 15.12 & $17.1-72.6$ & 79.9 & 34.3 & $27.7-153.7$ \\
\hline Minimum elevation from the $7 \mathrm{~mm}$ posterior BFS & -34.4 & 15.4 & $-75.0--14.1$ & -76.5 & 33.5 & $-160.9--29.3$ \\
\hline $\begin{array}{l}\text { Maximum minus minimum elevation from the } 7 \mathrm{~mm} \text { posterior } \\
\text { BFS }\end{array}$ & 67.2 & 29.2 & $34.0-142.8$ & 156.48 & 64.2 & $60.6-296.0$ \\
\hline Elevation of the thinnest point from the $8 \mathrm{~mm}$ anterior BFS & 2.6 & 1.5 & $0.0-6.0$ & 26.7 & 17.7 & $2.0-68.7$ \\
\hline Elevation of the thinnest point from the $8 \mathrm{~mm}$ posterior BFS & 4.1 & 4.7 & $-2.0-17.8$ & 58.4 & 39.0 & $6.3-143.4$ \\
\hline $\begin{array}{l}\text { Elevation of the thinnest point from the } 8 \mathrm{~mm} \text { posterior BFS } \\
\text { minus that of anterior BFS }\end{array}$ & 1.5 & 4.5 & $-5.0-14.0$ & 31.8 & 22.5 & $1.0-77.0$ \\
\hline Maximum elevation from the $8 \mathrm{~mm}$ posterior BFS & 51.4 & 23.3 & $21.0-107.9$ & 89.6 & 33.3 & $35.0-159.4$ \\
\hline Minimum elevation from the $8 \mathrm{~mm}$ posterior BFS & -43.0 & 18.3 & $-93.9--22.1$ & -90.9 & 41.1 & $-198.9--32.0$ \\
\hline $\begin{array}{l}\text { Maximum minus minimum elevation from the } 8 \mathrm{~mm} \text { posterior } \\
\text { BFS }\end{array}$ & 94.5 & 37.9 & $49.2-188.6$ & 180.5 & 71.4 & $67.6-351.8$ \\
\hline Elevation of the thinnest point from the $9 \mathrm{~mm}$ anterior BFS & 4.5 & 2.0 & $1.0-9.0$ & 34.0 & 21.5 & $6.0-90.7$ \\
\hline Elevation of the thinnest point from the $9 \mathrm{~mm}$ posterior BFS & 9.9 & 6.8 & $2.0-31.9$ & 72.7 & 43.9 & $11.0-171.8$ \\
\hline $\begin{array}{l}\text { Elevation of the thinnest point from the } 9 \mathrm{~mm} \text { posterior BFS } \\
\text { minus that of anterior BFS }\end{array}$ & 5.3 & 6.2 & $-2.0-24.9$ & 38.7 & 24.0 & $2.3-89.0$ \\
\hline Maximum elevation from the $9 \mathrm{~mm}$ posterior BFS & 76.5 & 28.16 & $43.1-145.9$ & 112.836 & 41.9722 & $48.7-212.7$ \\
\hline Minimum elevation from the $9 \mathrm{~mm}$ posterior BFS & -57.2 & 21.2 & $-124.0--33.2$ & -104.8 & 61.0 & $-248.4--40.3$ \\
\hline $\begin{array}{l}\text { Maximum minus minimum elevation from the } 9 \mathrm{~mm} \text { posterior } \\
\text { BFS }\end{array}$ & 133.7 & 45.7 & $77.6-284.5$ & 217.6 & 89.23 & $87.3-428.0$ \\
\hline
\end{tabular}


TABLE 1: Continued.

\begin{tabular}{|c|c|c|c|c|c|c|}
\hline \multirow{2}{*}{ Indices } & \multicolumn{3}{|c|}{ Normal } & \multicolumn{3}{|c|}{ Keratoconus stages 1 to 4} \\
\hline & Mean & SD & $95 \% \mathrm{CI}$ & Mean & SD & $95 \% \mathrm{CI}$ \\
\hline \multicolumn{7}{|l|}{ Using BFTE } \\
\hline Elevation of the thinnest point from the $7 \mathrm{~mm}$ posterior BFTE & 3.5 & 2.8 & $-1.0-10.0$ & 37.6 & 24.9 & $0.0-97.01$ \\
\hline Maximum elevation from the $7 \mathrm{~mm}$ posterior BFTE & 18.0 & 10.7 & $6.1-40.0$ & 71.3 & 54.8 & $17.7-225.6$ \\
\hline Minimum elevation from the $7 \mathrm{~mm}$ posterior BFTE & -25.8 & 8.6 & $-47.8--12.1$ & -72.8 & 39.6 & $-187.4--18.3$ \\
\hline $\begin{array}{l}\text { Maximum minus minimum elevation from the } 7 \mathrm{~mm} \text { posterior } \\
\text { BFTE }\end{array}$ & 43.8 & 16.3 & $23.0-80.3$ & 144.1 & 88.3 & $39.3-393.1$ \\
\hline Elevation of the thinnest point from the $8 \mathrm{~mm}$ posterior BFTE & 4.3 & 3.9 & $-2.0-13.0$ & 39.5 & 26.45 & $-3.0-104.0$ \\
\hline Maximum elevation from the $8 \mathrm{~mm}$ posterior BFTE & 43.9 & 22.2 & $11.1-114.8$ & 103.1 & 84.1 & $23.0-360.0$ \\
\hline Minimum elevation from the $8 \mathrm{~mm}$ posterior BFTE & -33.4 & 11.7 & $-62.9--16.1$ & -89.9 & 40.9 & $-185.1--29.0$ \\
\hline $\begin{array}{l}\text { Maximum minus minimum elevation from the } 8 \mathrm{~mm} \text { posterior } \\
\text { BFTE }\end{array}$ & 77.3 & 28.2 & $32.1-141.7$ & 192.9 & 114.2 & $68.0-557.4$ \\
\hline Elevation of the thinnest point from the $9 \mathrm{~mm}$ posterior BFTE & 3.6 & 5.6 & $-5.0-16.9$ & 38.7 & 30.8 & $-18.34-111.8$ \\
\hline Maximum elevation from the $9 \mathrm{~mm}$ posterior BFTE & 89.9 & 39.5 & $35.2-174.6$ & 170.8 & 152.9 & $39.6-665.5$ \\
\hline Minimum elevation from the $9 \mathrm{~mm}$ posterior BFTE & -42.1 & 15.5 & $-88.3--21.1$ & -114.5 & 50.2 & $-217.5--32.7$ \\
\hline $\begin{array}{l}\text { Maximum minus minimum elevation from the } 9 \mathrm{~mm} \text { posterior } \\
\text { BFTE }\end{array}$ & 132.0 & 43.3 & $65.2-231.4$ & 285.2 & 177.1 & $107.3-859.2$ \\
\hline \multicolumn{7}{|c|}{ Pachymetry indices } \\
\hline Apex thickness & 545.3 & 35.4 & $471.7-624.3$ & 482.5 & 39.4 & $384.3-573.4$ \\
\hline Central corneal thickness & 545.5 & 35.5 & $471.7-623.4$ & 491.4 & 35.3 & $408.9-573.4$ \\
\hline Thinnest point thickness & 543.5 & 35.7 & $471.7-622.3$ & 465.1 & 68.1 & $361.8-566.7$ \\
\hline PPI-Min & 0.543 & 0.1512 & $0.300-0.800$ & 1.504 & 0.8265 & $0.500-3.570$ \\
\hline PPI-Avg & 0.826 & 0.1365 & $0.600-1.100$ & 2.104 & 1.0454 & $0.900-4.967$ \\
\hline PPI-Max & 1.077 & 0.1716 & $0.800-1.400$ & 2.800 & 1.3960 & $1.232-6.705$ \\
\hline PPI-Max minus PPI-Min & 0.534 & 0.189 & $0.300-0.992$ & 1.296 & 0.763 & $0.400-3.835$ \\
\hline ART-Min & 1109.9 & 430.9 & $613.0-2044.2$ & 423.3 & 272.5 & $94.0-1045.4$ \\
\hline ART-Avg & 677.6 & 131.4 & $395.7-975.2$ & 276.6 & 138.2 & $75.7-616.9$ \\
\hline ART-Max & 517.8 & 90.6 & $326.6-697.0$ & 207.1 & 100.6 & $54.6-427.5$ \\
\hline Horizontal decentration of the thinnest point from the apex & 0.026 & 0.569 & $-0.948-1.019$ & -0.087 & 0.574 & $-0.940-0.827$ \\
\hline Vertical decentration of the thinnest point from the apex & -0.273 & 0.208 & $-0.639-0.214$ & -0.519 & 0.347 & $-1.394-0.110$ \\
\hline Diagonal decentration of the thinnest point from the apex & 0.618 & 0.240 & $0.202-1.088$ & 0.799 & 0.288 & $0.366-1.525$ \\
\hline
\end{tabular}

SD: standard deviation, 95\% CI: 95\% confidence interval, BFS: best-fit sphere, BFTE: best-fit toric ellipsoid, PPI-Min: Pachymetry Progression Index-Minimum, PPI-Avg: Pachymetry Progression Index-Average, PPI-Max: Pachymetry Progression Index-Maximum, ART-Min: Ambrósio’s Relational Thickness-Minimum, ART-Avg: Ambrósio’s Relational Thickness-Average, and ART-Max: Ambrósio’s Relational Thickness-Maximum.

2.1. Statistical Analysis. Data were collected and verified, and the compound indices were calculated using Microsoft Excel 2010 (Redmond, Washington, USA). Statistical analyses were performed using IBM SPSS Statistics (v19; Armonk, NY, USA) and Medcalc v11.1.1.0 (MedCalc, Belgium).

The following tests were performed: calculation of the mean, standard deviation (SD), unpaired $t$-test, sensitivity, specificity at different cut-off values, likelihood ratios, and area under the receiver operating characteristic curve (AUROC). AUROCs are compared using the DeLong method [13]. Values were considered statistically significant if $P$ value was less than 0.05 .

\section{Results}

3.1. Cases Characteristics. Average patients' age at the time of the initial Pentacam imaging was $29.2 \pm 8.8$ years (range 17.4 to 53.2 ) and $27.8 \pm 7.3$ years (range 14 to 44.4 ) in normal and KC groups, respectively. There was no statistically significant difference between the two groups $(P=0.253)$. The ratios between right and left eyes were $53: 50$ and $40: 33$ in normal and $\mathrm{KC}$ groups, respectively $(P=0.777)$.

The mean, SD, and 95\% confidence interval (CI) of each parameter are listed in Table 1.

Table 2 represents the AUROC of all tested indices when comparing normal corneas to all other grades of $\mathrm{KC}$ collectively, and Table 3 represents the ten indices with the highest AUROC. The AUROCs of these ten indices were statistically noninferior to each other $(P>0.05)$. Meanwhile, all other indices had statistically inferior AUROC to that of at least one of these ten indices. It is worth mentioning that none of the curvature indices was among these ten indices. 
TABLE 2: AUROC of all indices when differentiating all grades of KC collectively from normal corneas.

\begin{tabular}{|c|c|c|c|}
\hline Indices & AUROC & SEM & $95 \% \mathrm{CI}$ \\
\hline \multicolumn{4}{|c|}{ Curvature indices } \\
\hline \multicolumn{4}{|l|}{ Central $3 \mathrm{~mm}$} \\
\hline Mean radius of curvature of anterior $3 \mathrm{~mm}$ & 0.885 & 0.03 & 0.828 to 0.928 \\
\hline Mean radius of curvature of posterior $3 \mathrm{~mm}$ & 0.882 & 0.0307 & 0.824 to 0.925 \\
\hline Mean eccentricity of the anterior surface & 0.811 & 0.039 & 0.745 to 0.866 \\
\hline \multicolumn{4}{|l|}{ Using BFS } \\
\hline Radius of curvature of the $8 \mathrm{~mm}$ anterior BFS & 0.812 & 0.035 & 0.747 to 0.867 \\
\hline Radius of curvature of the $9 \mathrm{~mm}$ anterior BFS & 0.776 & 0.0374 & 0.707 to 0.835 \\
\hline Radius of curvature of the $7 \mathrm{~mm}$ posterior BFS & 0.841 & 0.0323 & 0.779 to 0.892 \\
\hline Radius of curvature of the $8 \mathrm{~mm}$ posterior BFS & 0.784 & 0.037 & 0.712 to 0.856 \\
\hline Radius of curvature of the $9 \mathrm{~mm}$ posterior BFS & 0.746 & 0.038 & 0.671 to 0.821 \\
\hline \multicolumn{4}{|l|}{ Using BFTE } \\
\hline Flattest radius of curvature of the $7 \mathrm{~mm}$ posterior BFTE & 0.829 & 0.0344 & 0.765 to 0.882 \\
\hline Steepest radius of curvature of the $7 \mathrm{~mm}$ posterior BFTE & 0.895 & 0.0298 & 0.840 to 0.936 \\
\hline Eccentricity of the $7 \mathrm{~mm}$ posterior BFTE & 0.757 & 0.0402 & 0.687 to 0.818 \\
\hline Flattest radius of curvature of the $8 \mathrm{~mm}$ posterior BFTE & 0.834 & 0.034 & 0.770 to 0.885 \\
\hline Steepest radius of curvature of the $8 \mathrm{~mm}$ posterior BFTE & 0.895 & 0.0298 & 0.840 to 0.936 \\
\hline Eccentricity of the $8 \mathrm{~mm}$ posterior BFTE & 0.757 & 0.0402 & 0.687 to 0.818 \\
\hline Flattest radius of curvature of the $9 \mathrm{~mm}$ posterior BFTE & 0.833 & 0.034 & 0.769 to 0.885 \\
\hline Steepest radius of curvature of the $9 \mathrm{~mm}$ posterior BFTE & 0.884 & 0.0313 & 0.827 to 0.927 \\
\hline Eccentricity of the $9 \mathrm{~mm}$ posterior BFTE & 0.757 & 0.0402 & 0.687 to 0.818 \\
\hline
\end{tabular}

Elevation indices

Elevation of the thinnest point from the $7 \mathrm{~mm}$ posterior BFS

Maximum elevation from the $7 \mathrm{~mm}$ posterior BFS

Minimum elevation from the $7 \mathrm{~mm}$ posterior BFS

Maximum minus minimum elevation from the $7 \mathrm{~mm}$ posterior BFS

Elevation of the thinnest point from the $7 \mathrm{~mm}$ posterior BFTE

Maximum elevation from the $7 \mathrm{~mm}$ posterior BFTE

Minimum elevation from the $7 \mathrm{~mm}$ posterior BFTE

Maximum minus minimum elevation from the $7 \mathrm{~mm}$ posterior BFTE

Elevation of the thinnest point from the $8 \mathrm{~mm}$ anterior BFS

Elevation of the thinnest point from the $8 \mathrm{~mm}$ posterior BFS

Maximum elevation from the $8 \mathrm{~mm}$ posterior BFS

Minimum elevation from the $8 \mathrm{~mm}$ posterior BFS

Maximum minus minimum elevation from the $8 \mathrm{~mm}$ posterior BFS

Elevation of the thinnest point from the $8 \mathrm{~mm}$ anterior BFS minus that of posterior BFS

Elevation of the thinnest point from the $8 \mathrm{~mm}$ posterior BFTE

Maximum elevation from the $8 \mathrm{~mm}$ posterior BFTE

Minimum elevation from the $8 \mathrm{~mm}$ posterior BFTE

Maximum minus Minimum elevation from the $8 \mathrm{~mm}$ posterior BFTE

Elevation of the thinnest point from the $9 \mathrm{~mm}$ anterior BFS

Elevation of the thinnest point from the $9 \mathrm{~mm}$ posterior BFS

Maximum elevation from the $9 \mathrm{~mm}$ posterior BFS

Minimum elevation from the $9 \mathrm{~mm}$ posterior BFS

Maximum minus minimum elevation from the $9 \mathrm{~mm}$ posterior BFS

Elevation of the thinnest point from the $9 \mathrm{~mm}$ anterior BFS minus that of posterior BFS

Elevation of the thinnest point from the $9 \mathrm{~mm}$ posterior BFTE

Maximum elevation from the $9 \mathrm{~mm}$ posterior BFTE

Minimum elevation from the $9 \mathrm{~mm}$ posterior BFTE

Maximum minus minimum elevation from the $9 \mathrm{~mm}$ posterior BFTE

\begin{tabular}{ccc}
0.971 & 0.0137 & 0.934 to 0.990 \\
0.916 & 0.0202 & 0.865 to 0.953 \\
0.908 & 0.0219 & 0.855 to 0.946 \\
0.923 & 0.0193 & 0.873 to 0.957 \\
0.953 & 0.0214 & 0.910 to 0.979 \\
0.944 & 0.0161 & 0.899 to 0.973 \\
0.91 & 0.0264 & 0.858 to 0.948 \\
0.949 & 0.0174 & 0.905 to 0.976 \\
0.968 & 0.018 & 0.930 to 0.989 \\
0.979 & 0.00837 & 0.945 to 0.995 \\
0.837 & 0.0304 & 0.775 to 0.889 \\
0.89 & 0.0255 & 0.834 to 0.932 \\
0.875 & 0.0272 & 0.817 to 0.920 \\
0.961 & 0.0139 & 0.920 to 0.984 \\
0.933 & 0.0264 & 0.885 to 0.965 \\
0.806 & 0.0342 & 0.739 to 0.861 \\
0.923 & 0.022 & 0.873 to 0.958 \\
0.912 & 0.0225 & 0.861 to 0.950 \\
0.979 & 0.0118 & 0.945 to 0.995 \\
0.977 & 0.0098 & 0.942 to 0.994 \\
0.772 & 0.0364 & 0.703 to 0.832 \\
0.865 & 0.0294 & 0.805 to 0.912 \\
0.832 & 0.0324 & 0.768 to 0.884 \\
0.952 & 0.0171 & 0.909 to 0.979 \\
0.904 & 0.0309 & 0.851 to 0.943 \\
0.671 & 0.0451 & 0.597 to 0.740 \\
0.934 & 0.0204 & 0.886 to 0.966 \\
0.887 & 0.0264 & 0.831 to 0.930 \\
\hline & &
\end{tabular}


TABLE 2: Continued.

\begin{tabular}{|c|c|c|c|}
\hline Indices & AUROC & SEM & $95 \% \mathrm{CI}$ \\
\hline \multicolumn{4}{|c|}{ Pachymetry indices } \\
\hline Apex thickness & 0.897 & 0.0268 & 0.842 to 0.938 \\
\hline Central corneal thickness & 0.878 & 0.0288 & 0.820 to 0.922 \\
\hline Thinnest point thickness & 0.915 & 0.0241 & 0.863 to 0.952 \\
\hline PPI-Min & 0.939 & 0.0208 & 0.893 to 0.969 \\
\hline PPI-Avg & 0.978 & 0.00973 & 0.944 to 0.994 \\
\hline PPI-Max & 0.987 & 0.00563 & 0.958 to 0.998 \\
\hline PPI-Max minus PPI-Min & 0.903 & 0.0246 & 0.850 to 0.943 \\
\hline ART-Min & 0.949 & 0.0183 & 0.905 to 0.976 \\
\hline ART-Avg & 0.976 & 0.0101 & 0.942 to 0.993 \\
\hline ART-Max & 0.987 & 0.00605 & 0.957 to 0.998 \\
\hline Horizontal decentration of the thinnest point from the apex & 0.558 & 0.0444 & 0.481 to 0.633 \\
\hline Vertical decentration of the thinnest point from the apex & 0.737 & 0.0397 & 0.666 to 0.801 \\
\hline Diagonal decentration of the thinnest point from the apex & 0.686 & 0.0403 & 0.612 to 0.754 \\
\hline
\end{tabular}

AUROC: area under the receiver operating characteristic curve. SEM: standard error of the mean. 95\% CI: 95\% confidence interval of the AUROC. BFS: best-fit sphere, BFTE: best-fit toric ellipsoid, PPI-Min: Pachymetry Progression Index-Minimum, PPI-Avg: Pachymetry Progression Index-Average, PPI-Max: Pachymetry Progression Index-Maximum, ART-Min: Ambrósio’s Relational Thickness-Minimum, ART-Avg: Ambrósio’s Relational Thickness-Average, and ART-Max: Ambrósio's Relational Thickness-Maximum.

TABLE 3: The indices with highest AUROC.

\begin{tabular}{|c|c|c|c|c|c|c|c|c|}
\hline Indices & AUROC & $95 \% \mathrm{CI}$ & Criterion & Sensitivity & Specificity & $\mathrm{LR}+$ & LR- & $\begin{array}{c}\text { AUROC } \\
\text { compared to } \\
\text { that of PPI-Max }\end{array}$ \\
\hline PPI-Max & 0.987 & 0.958 to 0.998 & $>1.4$ & 91.78 & 98.06 & 47.27 & 0.084 & \\
\hline ART-Max & 0.987 & 0.957 to 0.998 & $\leq 412$ & 97.26 & 93.2 & 14.31 & 0.029 & $P=0.880$ \\
\hline $\begin{array}{l}\text { Elevation of the thinnest point from } \\
\text { the } 9 \mathrm{~mm} \text { anterior BFS }\end{array}$ & 0.979 & 0.945 to 0.995 & $>7$ & 95.89 & 92.23 & 12.35 & 0.045 & $P=0.501$ \\
\hline $\begin{array}{l}\text { Elevation of the thinnest point from } \\
\text { the } 8 \mathrm{~mm} \text { anterior BFS }\end{array}$ & 0.968 & 0.930 to 0.989 & $>5$ & 91.78 & 96.12 & 23.63 & 0.086 & $P=0.288$ \\
\hline $\begin{array}{l}\text { Elevation of the thinnest point from } \\
\text { the } 8 \mathrm{~mm} \text { posterior BFS }\end{array}$ & 0.979 & 0.945 to 0.995 & $>20$ & 86.3 & 100 & N/A & 0.14 & $P=0.229$ \\
\hline $\begin{array}{l}\text { Elevation of the thinnest point from } \\
\text { the } 7 \mathrm{~mm} \text { posterior BFS }\end{array}$ & 0.971 & 0.934 to 0.990 & $>10$ & 87.67 & 100 & N/A & 0.12 & $P=0.217$ \\
\hline $\begin{array}{l}\text { Elevation of the thinnest point from } \\
\text { the } 9 \mathrm{~mm} \text { posterior BFS }\end{array}$ & 0.977 & 0.942 to 0.994 & $>22$ & 89.04 & 96.12 & 22.93 & 0.11 & $P=0.199$ \\
\hline PPI-Avg & 0.978 & 0.944 to 0.994 & $>1.1$ & 87.67 & 98.06 & 45.15 & 0.13 & $P=0.147$ \\
\hline $\begin{array}{l}\text { Elevation of the thinnest point from } \\
\text { the } 7 \mathrm{~mm} \text { posterior BFTE }\end{array}$ & 0.953 & 0.910 to 0.979 & $>10$ & 87.67 & 99.03 & 90.3 & 0.12 & $P=0.104$ \\
\hline ART-Avg & 0.976 & 0.942 to 0.993 & $\leq 496$ & 94.52 & 94.17 & 16.23 & 0.058 & $P=0.089$ \\
\hline All other tested indices & & & & & & & & $P<0.05$ \\
\hline
\end{tabular}

AUROC: area under the receiver operating characteristic curve. 95\% CI: 95\% confidence interval of the AUROC. LR+: likelihood ratio of positive results. LR-: likelihood ratio of negative results. $P$ from PPI-Max: probability of chance that the AUROC is less than that of PPI-Max. PPI-Max: Pachymetry Progression Index-Maximum, ART-Max: Ambrósio’s Relational Thickness-Maximum, BFS: best-fit sphere, PPI-Avg: Pachymetry Progression Index-Average, BFTE: bestfit toric ellipsoid, and ART-Avg: Ambrósio's Relational Thickness-Average.

Among the examined patients of both groups, pachymetry progression indices showed the highest AUROC accuracy among the evaluated parameters, where the Pachymetry Progression Index-Maximum (PPI-Max) and Ambrósio's Relational Thickness-Maximum (ART-Max) had the same highest AUROC (0.987). The best cut-off for PPI-Max was (>1.4 with sensitivity $91.78 \%$ and specificity $98.06 \%$ ) and for
ART-Max was ( $\leq 412$ with sensitivity $97.26 \%$ and specificity 93.2\%).

The further analysis of the indices according to patients' grouping (based on TKC grading) showed that the mean values of all indices were significantly different when comparing group $2 c$ corneas and normal corneas $(P<0.05)$, with the only exception of horizontal decentration of the 
TABLE 4: Scheimpflug imaging indices differentiating between GROUP 2a KC and normal corneas.

\begin{tabular}{lccc}
\hline Indices & AUROC & SEM & $95 \%$ CI \\
\hline PPI-Max & 0.690 & 0.038 & 0.615 to 0.765 \\
ART-Max & 0.690 & 0.038 & 0.615 to 0.764 \\
PPI-Max minus PPI-Min & 0.687 & 0.055 & 0.579 to 0.796 \\
Central corneal thickness & 0.683 & 0.048 & 0.590 to 0.776 \\
$\begin{array}{l}\text { Diagonal decentration of the thinnest } \\
\text { point from the apex }\end{array}$ & 0.674 & 0.062 & 0.552 to 0.795 \\
\hline
\end{tabular}

AUROC: area under the receiver operating characteristic curve, SEM: standard error of the mean, 95\% CI: 95\% confidence interval of the AUROC, PPI-Max: Pachymetry Progression Index-Maximum, ART-Max: Ambrósio’s Relational Thickness-Maximum, and PPI-Min: Pachymetry Progression Index-Minimum.

thinnest point from the apex $(P=0.292)$. Meanwhile, only three indices were statistically insignificant when comparing their values in group $2 \mathrm{~b}$ keratoconic corneas against group 1 normal corneas (radius of curvature of the $9 \mathrm{~mm}$ posterior BFS $(P=0.061)$ and maximum elevated point of the $9 \mathrm{~mm}$ posterior BFTE $(P=0.361)$, in addition to horizontal decentration of the thinnest point from the apex $(P=0.150)$. Contrarily, only five indices' values were statistically significant in discriminating group 2a KC corneas from normal corneas, as shown in Table 4.

\section{Discussion}

Placido-based systems rely on the analysis of a reflected image. This cannot provide data from the posterior corneal surface [6]. Additionally, without information about the posterior corneal surface, complete pachymetric evaluation is not possible [14]. Ultrasonic central pachymetry measures one point, which is not necessarily the thinnest point, and does not reflect the overall thickness profile [15]. Moreover, the posterior corneal surface is appreciated as a sensitive indicator of corneal ectasia and can often be abnormal in spite of a still normal anterior corneal surface. While the corneal refractive power is largely determined by the anterior surface, the evaluation of anatomical properties of the cornea is at least equally dependent upon both surfaces [14]. Therefore, corneal tomography parameters assessment appeared to be essential in detecting KC.

With the appearance of many $\mathrm{KC}$ indices and suggestion of various best-fit reference surfaces [6], the goal of our study was to evaluate the accuracy (including both sensitivity and specificity) of such indices with the use of different reference surfaces, at different diameters, and correlate these parameters to TKC grading. Some previous studies used cyclic evidence that stated that a certain index was accurate based on differentiating $\mathrm{KC}$ from normal cornea using certain criteria closely related to this same index. Our study adds the long follow-up (6 years) as a more robust evidence. If a cornea was falsely diagnosed as normal and underwent LASIK, it would develop ectasia in such a long follow-up period. On the other side, a KC diagnosis can be confirmed if it spontaneously progressed over time.

The inclusion of every single possible index is out of the scope of this study. The included indices are those mentioned individually in other studies or those we thought they might be significant. On evaluating BFTE and smaller best-fit surface $(7 \mathrm{~mm})$, we focused on the posterior rather than the anterior corneal surface, being the site for primary subclinical tomographic changes, preceding the anterior surface [16-19].

Curvature-based indices derived from both anterior and posterior surfaces were evaluated and analysed. According to the ROC curves, all evaluated curvature indices had statistically significant less AUROC for diagnosing KC than most of elevation and pachymetry indices. Previous reports demonstrated similar results [20-22]. Despite being the least significant in diagnosing $\mathrm{KC}$, they have been considered as important criteria in KC and after LASIK ectasia follow-up $[23,24]$.

In this study, where we only analysed data from good quality scans with corneal surface area more than $9 \mathrm{~mm}$, analysis of elevation indices showed that posterior surface elevation (PE) from $8 \mathrm{~mm}$ BFS and anterior surface elevation (AE) from $9 \mathrm{~mm}$ BFS had the highest accuracy, with AUROC (0.979) for both, followed by PE from 7 and $9 \mathrm{~mm}$ BFS (AUROC $=0.977$ and 0.971 , resp.). $\mathrm{AE}$ and $\mathrm{PE}$ from reference best-fit surfaces did not differ in their accuracy with various diameters $(7,8$, and $9 \mathrm{~mm})$. Correia et al. reported that PE from both $8 \mathrm{~mm}$ BFS and BFTE had highest AUROC $(0.983$ and 0.986, resp.) [20]. de Sanctis et al. [25] evaluated the sensitivity and specificity of $\mathrm{PE}$ from $9 \mathrm{~mm} \mathrm{BFS}$ in discriminating normal corneas from $\mathrm{KC}$. The AUROC analyses showed high overall predictive accuracy of PE for KC (AUROC 0.99). They concluded that PE very effectively discriminates KC from normal corneas, but its efficacy is lower for subclinical KC, and thus data concerning PE should not be used alone to stratify patients with this condition.

We enrolled in our work the elevation indices of the thinnest point from both BFS and BFTE. The latter was evaluated in literature and showed higher predictive accuracy in diagnosing $\mathrm{KC}$ and forme fruste $\mathrm{KC}$ compared to BFS, especially with a fixed eccentricity. A possible explanation for its diagnostic superiority in ectatic corneas is that its central vaulting can determine whether the corneal pattern is associated with an atypical condition, a true corneal disease, or an artifact of alignment or processing $[16,20]$.

According to our study results, parameters that had the highest AUROC for diagnosing KC were the PPI-Max at cutoff value of $>1.4$ and the ART-Max at cut-off value of $\leq 412$ ( 0.987 for both). These results were comparable to those of Ambrósio Jr. et al. [1] who reported that the most sensitive 
parameters were ART-Avg and ART-Max (AUROC $=0.987$ and 0.983 , resp.).

Our study investigated the accuracy of various tomographic indices in relation to TKC grading [26]. We found that most of the tested indices had significantly different values between each of grades 2 to 4 (moderate and/or severe) $\mathrm{KC}$ and normal corneas. In 2012, Ishii and his coworkers [27] investigated the severity of KC in terms of corneal elevation differences, and they correlated the data with AmslerKrumeich classification of KC. They observed that cases of higher elevation differences in the anterior and posterior corneal surfaces were staged higher in Amsler-Krumeich classification $(P<0.001)$ and concluded that anterior and posterior corneal surface height data obtained by elevationbased tomography provide useful information in improving $\mathrm{KC}$ diagnostic accuracy and in grading the severity of KC. Their results are comparable to ours in the accuracy of elevation indices; however, they did not furtherly investigate other indices.

Furthermore, Kamiya and his colleagues [28] evaluated corneal elevation, pachymetry, and keratometry in KC eyes according to the clinical stage of the disease, with respect to each stage of Amsler-Krumeich classifications. They detected that PE (0.980) and AE (0.977) showed the highest AUROCs, followed by $K \max$ value (0.941), percentage thickness increase (PTI) $2 \mathrm{~mm}$ (0.931), PTI $4 \mathrm{~mm}$ (0.927), and PPIMax (0.927). Their results are highly comparable to ours in AUROC of indices. They observed that $\mathrm{AE}$ and $\mathrm{PE}$ measurements tended to have a higher accuracy at the earlier stages of KC, when compared to other studied parameters; hence, they concluded that elevation difference measurements might provide useful information for improving the diagnostic accuracy of KC, especially in the early stage. These results contradict ours, where we found that the indices with statistically significant high AUROC when comparing grade $1 \mathrm{KC}$ to normal corneas were PPI-Max, ART-Max, PPIMax minus PPI-Min, central corneal thickness, and diagonal decentration of the thinnest point from the apex.

Our findings justify the modifications in the newer software versions that incorporated "ART-Max" and "diagonal decentration of the thinnest point from the apex," being sensitive indices for early KC detection. As regards the hardware changes, we also recommend similar studies on Pentacam HR, because the current one was on Allegro Oculyzer I Pentacam. Randleman et al. [29] reported that, for refractive surgical screening, regular and high-resolution Scheimpflug imaging devices generated different objective values and the two devices are not interchangeable.

\section{Conclusion}

The accuracy of different curvature, elevation, and pachymetry-based indices using various reference shapes of different diameters was evaluated, in relation to TKC grading. To the best of our knowledge, this is the first study to confirm clinical and topographical observations by a long follow-up period of 6 years to ascertain the diagnosis by time evidence. Generally, to discriminate all $\mathrm{KC}$ grades from normal, ten pachymetry and elevation-based indices were significantly more accurate than other indices, having higher AUROC. Five of them had statistically significant high AUROC when comparing early $\mathrm{KC}$ to normal corneas.

\section{Competing Interests}

There are no competing interests of any of the authors with any establishment having a relation to this present work.

\section{Acknowledgments}

Sherine S. Wahba and Maged M. Roshdy received travel support by Alcon Laboratories Inc., USA, to attend ESCRS Congress.

\section{References}

[1] R. Ambrósio Jr., A. L. C. Caiado, F. P. Guerra et al., "Novel pachymetric parameters based on corneal tomography for diagnosing keratoconus," Journal of Refractive Surgery, vol. 27, no. 10, pp. 753-758, 2011.

[2] M. W. Belin, D. Litoff, S. J. Strods, S. S. Winn, and R. S. Smith, "The PAR technology corneal topography system," Refractive and Corneal Surgery, vol. 8, no. 1, pp. 88-96, 1992.

[3] M. K. Smolek, "What is keratoconus?" in Modern Management of Keratoconus, B. S. Boxer Wachler, Ed., p. 3, Jaypee Brothers Medical Publishers, New Delhi, India, 1st edition, 2008.

[4] R. Ambrósio, D. G. Dawson, and M. W. Belin, "Association between the percent tissue altered and post-laser in situ keratomileusis ectasia in eyes with normal preoperative topography," American Journal of Ophthalmology, vol. 158, no. 6, pp. 1358-1359, 2014.

[5] D. Z. Reinstein, T. J. Archer, and M. Gobbe, "Corneal epithelial thickness profile in the diagnosis of keratoconus," Journal of Refractive Surgery, vol. 25, no. 7, pp. 604-610, 2009.

[6] M. W. Belin, S. S. Khachikian, and R. Ambrosio, "Understanding elevation based topography: how elevation data is displayed," in Elevaton Based Corneal Tomography, M. W. Belin, S. S. Khachikian, and R. Ambrosio, Eds., chapter 3, pp. 2546, Jaypee-Highlights Medical Publishers, Inc. Panama, 2nd edition, 2012.

[7] S. Ramamurthy, J. C. Reddy, and V. Jhanji, "Topography and tomography in the diagnosis of corneal ectasia," Expert Review of Ophthalmology, vol. 10, no. 3, pp. 215-228, 2015.

[8] G. Labiris, A. Giarmoukakis, A. Koutras, T. Chrysochou, H. Sideroudi, and V. P. Kozobolis, "Clinical usefulness of bestfit toric ellipsoid reference body in ectatic and crosslinked corneas," Journal of Cataract \& Refractive Surgery, vol. 41, no. 4, pp. 889-891, 2015.

[9] I. Kovács, K. Miháltz, M. Ecsedy, J. Németh, and Z. Z. Nagy, "The role of reference body selection in calculating posterior corneal elevation and prediction of keratoconus using rotating Scheimpflug camera," Acta Ophthalmologica, vol. 89, no. 3, pp. e251-e256, 2011.

[10] G. Labiris, A. Giarmoukakis, A. Rampotas et al., "Variability of different reference bodies in normal, keratoconus, and collagen crosslinked corneas," European Journal of Ophthalmology, vol. 25, no. 6, pp. 468-473, 2015. 
[11] M. K. Smolek and B. S. Boxer Wachler, "Corneal topography," in Modern Management of Keratoconus, B. S. Boxer Wachler, Ed., p. 35, Jaypee, New Delhi, India, 1st edition, 2008.

[12] Allegro Oculyzer 1074 user manual en/Rev.5/10-11-02 Item No.: 6654 2001, WaveLight GmbH, Germany.

[13] E. R. DeLong, D. M. DeLong, and D. L. Clarke-Pearson, "Comparing the areas under two or more correlated receiver operating characteristic curves: a nonparametric approach," Biometrics, vol. 44, no. 3, pp. 837-845, 1988.

[14] P. S. Binder, R. L. Lindstrom, R. D. Stulting et al., "Keratoconus and corneal ectasia after LASIK," Journal of Cataract and Refractive Surgery, vol. 31, no. 11, pp. 2035-2038, 2005.

[15] M. W. Belin and S. S. Khachikian, "New devices and clinical implications for measuring corneal thickness," Clinical \& Experimental Ophthalmology, vol. 34, no. 8, pp. 729-731, 2006.

[16] H. Sideroudi, G. Labiris, A. Giarmoukakis, N. Bougatsou, and V. Kozobolis, "Contribution of reference bodies in diagnosis of keratoconus," Optometry and Vision Science, vol. 91, no. 6, pp. 676-681, 2014.

[17] T. C. Y. Chan, D. Liu, M. Yu, and V. Jhanji, "Longitudinal evaluation of posterior corneal elevation after laser refractive surgery using swept-source optical coherence tomography," Ophthalmology, vol. 122, no. 4, pp. 687-692, 2015.

[18] B. Wang, Z. Zhang, R. K. Naidu et al., "Comparison of the change in posterior corneal elevation and corneal biomechanical parameters after small incision lenticule extraction and femtosecond laser-assisted LASIK for high myopia correction," Contact Lens \& Anterior Eye, vol. 39, no. 3, pp. 191-196, 2016.

[19] A. Saad and D. Gatinel, "Screening for keratoconus: lack of a scientific base regarding the global consensus?" Cornea, vol. 34, no. 11, pp. e33-e34, 2015.

[20] F. F. Correia, I. Ramos, B. Lopes et al., "Topometric and tomographic indices for the diagnosis of keratoconus," International Journal of Keratoconus and Ectatic Corneal Diseases, vol. 1, no. 2, pp. 92-99, 2012.

[21] R. Ambrósio Jr., L. P. Nogueira, D. L. Caldas et al., "Evaluation of corneal shape and biomechanics before LASIK," International Ophthalmology Clinics, vol. 51, no. 2, pp. 11-38, 2011.

[22] M. W. Belin and R. Ambrósio Jr., "Corneal ectasia risk score: statistical validity and clinical relevance," Journal of Refractive Surgery, vol. 26, no. 4, pp. 238-240, 2010.

[23] R. L. Epstein, Y.-L. Chiu, and G. L. Epstein, "Pentacam HR criteria for curvature change in keratoconus and postoperative LASIK ectasia," Journal of Refractive Surgery, vol. 28, no. 12, pp. 890-894, 2012.

[24] A. J. Kanellopoulos and G. Asimellis, "Revisiting keratoconus diagnosis and progression classification based on evaluation of corneal asymmetry indices, derived from scheimpflug imaging in keratoconic and suspect cases," Clinical Ophthalmology, vol. 7, pp. 1539-1548, 2013.

[25] U. de Sanctis, C. Loiacono, L. Richiardi, D. Turco, B. Mutani, and F. M. Grignolo, "Sensitivity and specificity of posterior corneal elevation measured by pentacam in discriminating keratoconus/subclinical keratoconus," Ophthalmology, vol. 115, no. 9, pp. 1534-1539, 2008.

[26] J. H. Krumeich, J. Daniel, and A. Knalle, "Live-epikeratophakia for keratoconus," Journal of Cataract and Refractive Surgery, vol. 24, no. 4, pp. 456-463, 1998.

[27] R. Ishii, K. Kamiya, A. Igarashi, K. Shimizu, Y. Utsumi, and T. Kumanomido, "Correlation of corneal elevation with severity of keratoconus by means of anterior and posterior topographic analysis," Cornea, vol. 31, no. 3, pp. 253-258, 2012.
[28] K. Kamiya, R. Ishii, K. Shimizu, and A. Igarashi, "Evaluation of corneal elevation, pachymetry and keratometry in keratoconic eyes with respect to the stage of Amsler-Krumeich classification," British Journal of Ophthalmology, vol. 98, no. 4, pp. 459463, 2014.

[29] J. B. Randleman, J. Akhtar, M. J. Lynn et al., "Comparison of objective and subjective refractive surgery screening parameters between regular and high-resolution Scheimpflug imaging devices," Journal of Cataract \& Refractive Surgery, vol. 41, no. 2, pp. 286-294, 2015. 


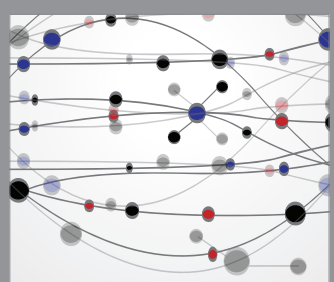

The Scientific World Journal
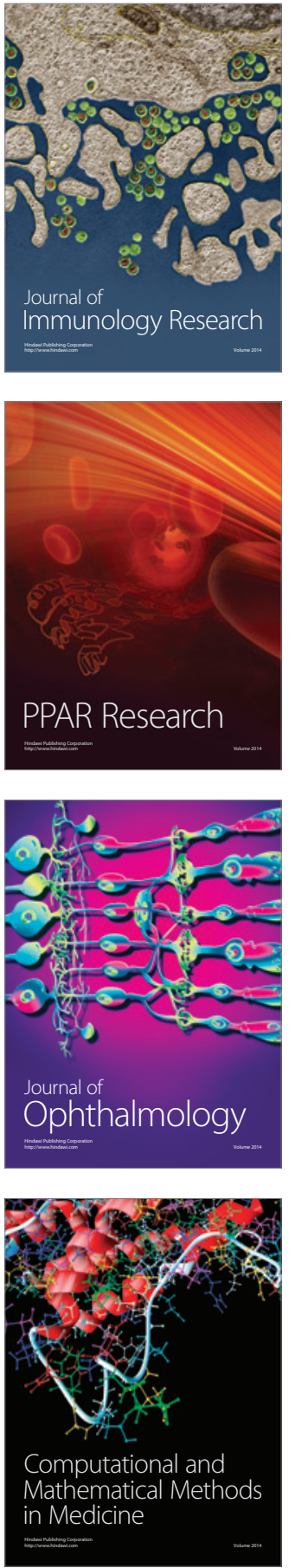

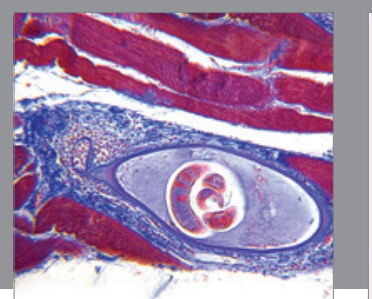

Gastroenterology Research and Practice

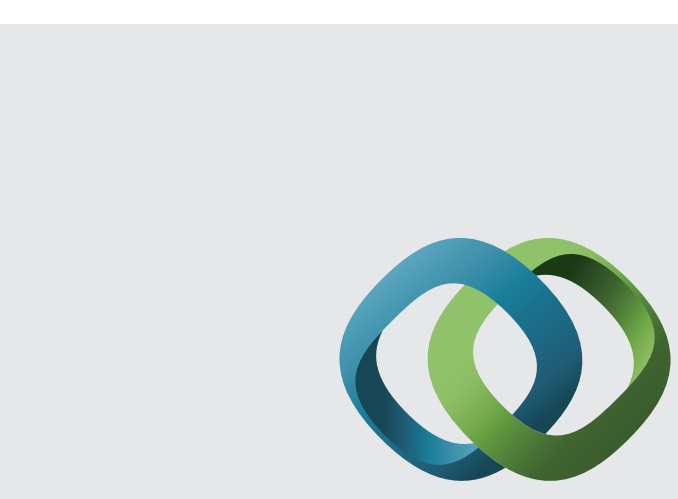

\section{Hindawi}

Submit your manuscripts at

http://www.hindawi.com
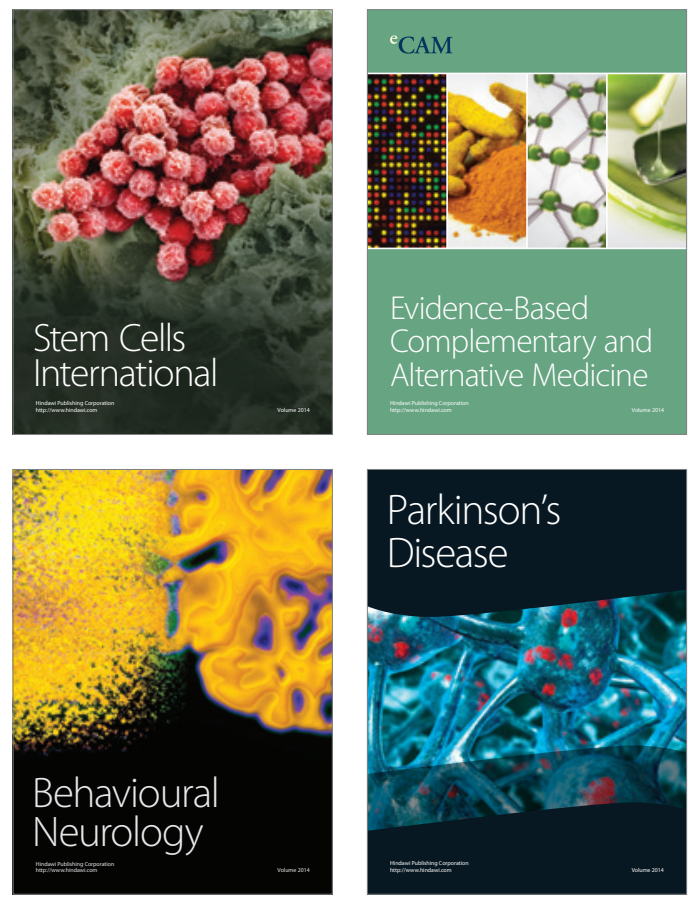
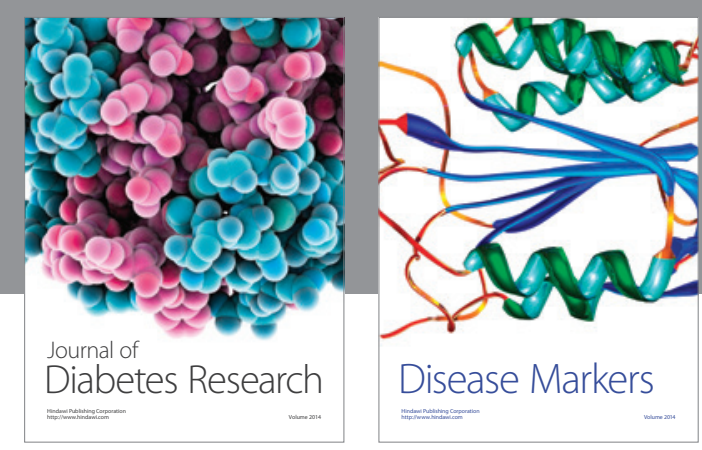

Disease Markers
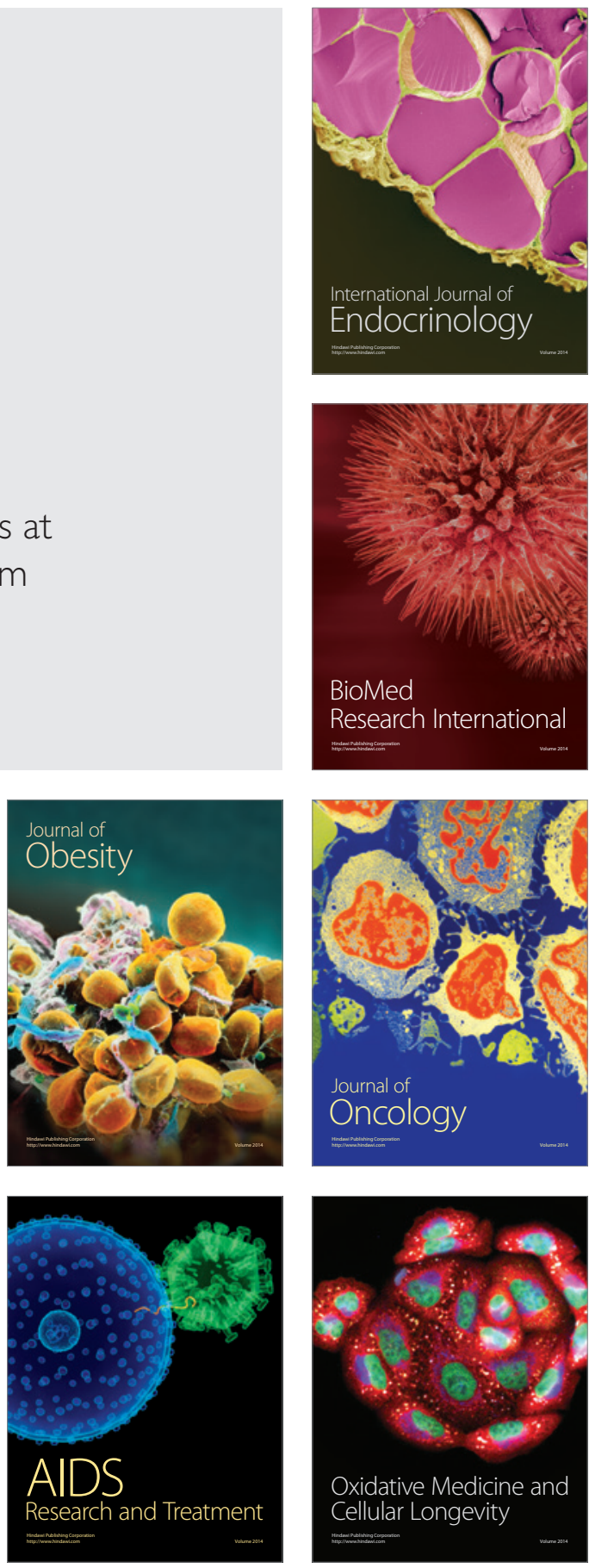